\title{
The Resource-Saving Technology of Aluminum Nitride Obtaining During Combustion of Aluminum Nanopowder in Air
}

\author{
Alexander Ilyin ${ }^{1, *}$, Andrei Mostovshchikov ${ }^{1}$, and Liudmila Root $^{1}$ \\ ${ }^{1}$ Tomsk Polytechnic University, 634050 Tomsk, Russia
}

\begin{abstract}
The resource-saving technology of aluminum nitride obtaining during the combustion of aluminum nanopowder in air has been analyzed in the article. The investigation of the crystal phases of aluminum nanopowder combustion products obtained under the magnetic field exposure has been made. The experimental results showed the increase of aluminum nitride content up to $86 \mathrm{wt} . \%$ in comparison with the aluminum nitride content in combustion products without any exposure. The mechanism of aluminum nitride formation and stabilization in air was due to the oxygen molecules deactivation by light emission during combustion.
\end{abstract}

\section{Introduction}

It is known that aluminium nitride has the following unique properties: a high thermal conductivity $\left(\lambda>250 \mathrm{~W} / \mathrm{m}^{2} \cdot \mathrm{s}\right)$ and low electrical conductivity $\left(\rho>10^{13} \mathrm{Ohm} \cdot \mathrm{m}\right)[1]$. At the same time, the technology of aluminum nitride synthesis is complicated by the absence of the liquid phase during heating (i.e. above $2400{ }^{\circ} \mathrm{C}$ aluminum nitride evaporates). Therefore, the technology of aluminum nitride production includes the stages of $\gamma-\mathrm{Al}_{2} \mathrm{O}_{3}$ with the coke prolonged heating at $1600-1700{ }^{\circ} \mathrm{C}$, grinding the product, and its re-nitriding. The sintering of items made from aluminum nitride is realized using a hot pressing method in the high-purity nitrogen medium [2,3]. The synthesis of aluminum nitride is also possible by the combustion of aluminum powder in pure nitrogen medium [4-6].

It was experimentally established that as the result of combustion of the powdered aluminum in air more than $60 \mathrm{wt}$. \% of aluminium nitride is stabilized in the final combustion products [7-10]. Even the first investigations of aluminum nanopowder combustion [11] showed, that the combustion of relatively small samples (less than $0.5 \mathrm{~g}$ ) took place in a single step $\left(600-800{ }^{\circ} \mathrm{C}\right)$, during which aluminum nitride did not form. If the sample mass of nanopowder exceeded $1 \mathrm{~g}$, the low-temperature combustion stage exceeded to the high-temperature stage $\left(2200-2400{ }^{\circ} \mathrm{C}\right)$, accompanied by a bright irradiation in the visible and infrared spectral ranges. This combustion stage passed in the thermal explosion mode. The combustion rate increased with the sample mass raise. The combustion conditions approximated adiabatic as a result of temperature and luminosity increase of the $\mathrm{Al}-\mathrm{O}_{2}-\mathrm{N}_{2}$ burning system. When the sample weight reached $15 \mathrm{~g}$, the

* Corresponding author: ilyin@tpu.ru 
aluminum nitride yield increased up to $66 \%$, taking into account that even at 10-12 g the growth of aluminum nitride yield in aluminum nanopowder combustion products in air slowed down. Furthermore, it was established in early studies that high-power exposure on the aluminium nanopowder during the combustion increases the thermal effect of its oxidation [12-14]. This is a promising trend for increasing the burning power of the aluminum nanopowder that can be also used to raise the aluminum nitride yield in the combustion synthesis [15].

The aim of this work was to improve the resource efficiency of aluminum nitride synthesis by increasing the yield of aluminum nitride in aluminum nanopowder combustion products under the magnetic field exposure.

\section{Experimental procedure}

The aluminum nanopowder obtained by the wire electrical explosion in argon, has been studied. The aluminum nanopowder was obtained with the help of UDP-4G machine, developed in National Research Tomsk Polytechnic University [16]. The concept scheme of the machine for nanopowders production is shown in Fig. 1.

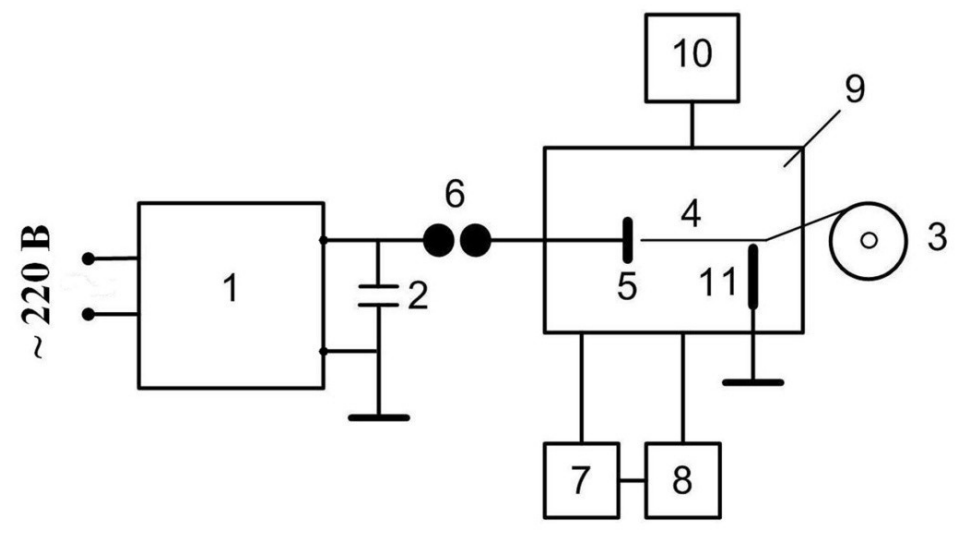

Fig. 1. The concept scheme of the wire electrical explosion machine for nanopowders production.

The machine operates in the following way. The capacitive energy storage (2) is charged from a high voltage power supply (1). The wire feeder (3) automatically moves the wire segment being exploded (4) to the inter-electrode gap. When the wire reaches the high-voltage electrode (5), the commutator (6) operates. The capacitive energy storage discharges on the wire segment between the high-voltage electrode and the ground electrode (11), as the result, the wire segment explodes. The obtained powder is collected in the powder collector (7). The gas purified from the powder is fed back into the explosion chamber (9) by means of the gas circulation system (8). The volume of the explosion chamber (9) before exploitation is evacuated and then filled with the required gas. These functions are performed by the gas supply system (10).

Aluminum nanopowder obtained in argon is pyrophoric, i.e. it ignites when exposed to air. It was characterized by the following activity parameters: oxidation start temperature $450{ }^{\circ} \mathrm{C}$; oxidation degree $-63.8 \%$; maximal oxidation rate $-0.13 \mathrm{wt} . \% / \mathrm{deg}$, specific heat effect $-4995 \mathrm{~J} / \mathrm{g}$. The particle size distribution was close to the regular-logarithmic with a maximum of $120 \mathrm{~nm}$. The content of the metallic impurities in nanopowder did not exceed 1.0 wt. $\%$ [17].

Alundum pot was used for the experiments on aluminum nanopowder combustion. The combustion of some nanopowder samples was performed in the conical shaped form on polycor or duralumin base in air, as well. To conduct experiments on combustion the 
optimal sample weight of $3 \mathrm{~g}$ was chosen, since at the weight less than $0.5 \mathrm{~g}$ the combustion process took place in a single step, and at the weight of $4 \mathrm{~g}$ the thermal destruction of alundum pots occurred. The process of aluminum nanopowder combustion in air occurred in two stages. On the first stage, after ignition, the heat waves spread over the sample, the temperature maximum reached $600-800{ }^{\circ} \mathrm{C}$. After that the burning process accelerated and passed in the thermal explosion mode, accompanied by the temperature rise up to 2000 $2100{ }^{\circ} \mathrm{C}$ (on the base and in the pot, correspondingly). The temperature pyrometric measurements were realized at the Laboratory of thermal control methods (Laboratory no. 34) of National Research Tomsk Polytechnic University by the thermal imager ThermaCam P65 HS.

To determine the influence of a homogeneous magnetic field on the morphology of combustion products the samples were ignited in the pot between the poles of a magnet $(0.34 \mathrm{~T})$. And for determination the influence of an inhomogeneous magnetic field the samples were ignited between two magnets each of the same induction $(0.37 \mathrm{~T})$.

To determine the influence of a constant electric field on the morphology of combustion products the samples were ignited between the plates of air-dielectric capacitor; the field potential gradient in the capacitor was $10 \mathrm{kV} / \mathrm{m}$.

In order to establish the sequence of crystalline phases formation during the combustion of aluminum nanopowder in air, the equipment of the precision diffraction analysis center with the electron storage ring VEPP-3 of NPI n. a. G. I. Budker SB RAS (Novosibirsk, Russia) was used. The synchrotron radiation with a wavelength of $0.10173 \mathrm{~nm}$ was focused on the compacted sample surface. After initiation of the combustion process, the diffraction patterns were recorded by a single-axis detector OD-3M, which simultaneously detects radiation in the range of angles about $60 \mathrm{grad}$. The detector was set to the angular range from 30 to 90 grad. As only in this range the reflections of maximum intensity from the crystalline phases, formation of which is possible by aluminum nanopowder combustion, are located. The time of one frame making was $0.3 \mathrm{~s}$ that allowed getting 200 shots in a burning time of $60 \mathrm{~s}[10]$.

\section{Experimental results and discussion}

As a result of aluminum nanopowder combustion in a constant magnetic field $(0.34 \mathrm{~T})$ in alundum pot the output of aluminum nitride was $83 \mathrm{wt}$. \%. Due to the additional thermal insulation during the combustion in the pot a higher temperature and luminosity were observed. 


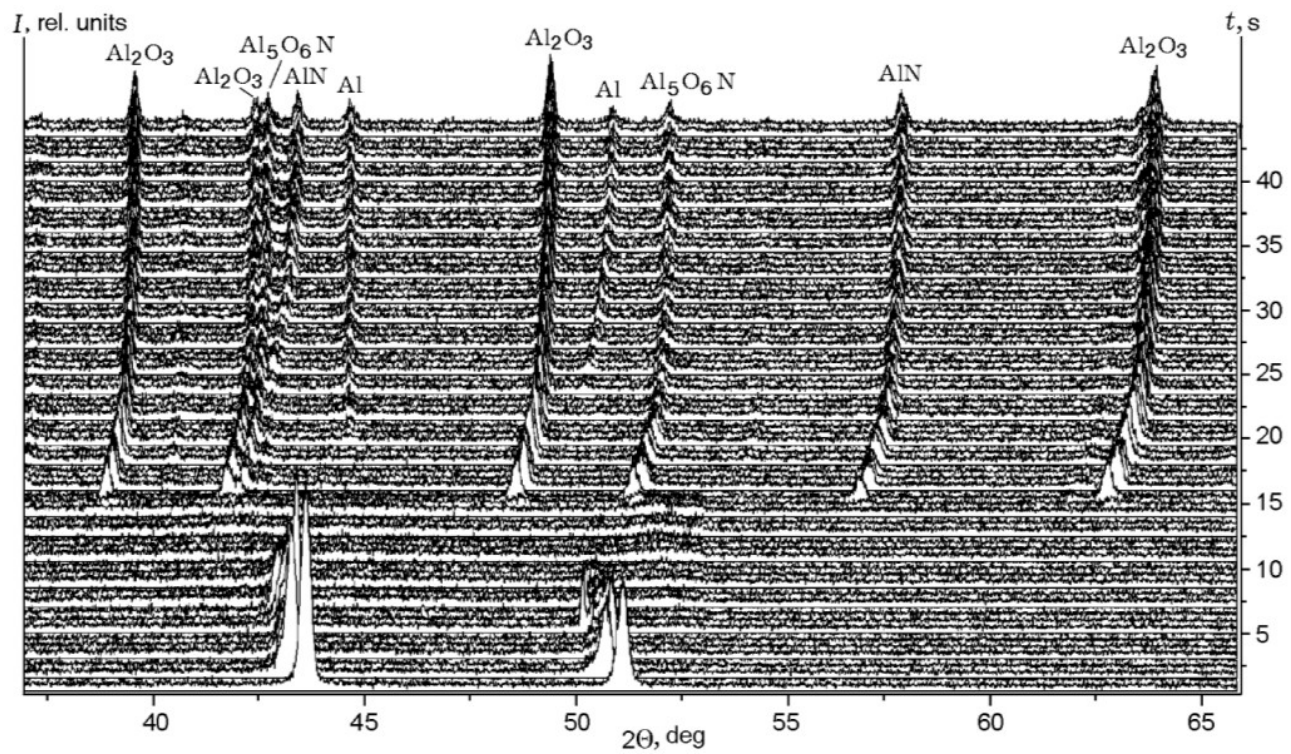

Fig. 2. Time scan of diffractograms of the compacted aluminum nanopowder surface while combustion.

When the compacted aluminum nanopowder (porosity of 66 vol. \%) was combusted in the field of synchrotron radiation $(\lambda=0,10173 \mathrm{~nm})$, an average of $86 \mathrm{wt} . \%$ of aluminum nitride was stabilized in the combustion products. According to the diffraction patterns, one of the mechanisms of the crystalline phase of aluminum nitride formation (Fig.2) is the consistent replacement of oxygen with nitrogen in aluminium oxide $\left(\mathrm{Al}_{2} \mathrm{O}_{3}+\mathrm{N}_{2} \rightarrow \mathrm{Al}_{5} \mathrm{O}_{6} \mathrm{~N}\right.$ $+\mathrm{N}_{2} \rightarrow$ AlN).

The formation of the independent crystalline phase of aluminum nitride involves the reaction of aluminum with nitrogen only at a certain stage of the combustion process. Nonparticipation of oxygen in aluminum oxidation can be explained by the deactivation of the oxygen molecules under the electromagnetic radiation exposure, namely, due to the photochemical transition of paramagnetic biradical $\mathrm{O}{ }^{*} \quad$ into a stable inactive molecule $\mathrm{O} \equiv \mathrm{O}[18]$.

Thus, the realization of the combustion process in a limited volume (pot) and in the magnetic field increased the yield of aluminum nitride in the combustion products 1.5-2 times, that results in the increase of the resource efficiency of the developed technology.

The research is supported by the Russian Foundation for Basic Research, project No 15-0305385 .

\section{References}

1. R. Bachelard, P. Joubert, Mater. Sci. Eng. 109A (1989)

2. G. Selvaduray, L. Sheet, Mater. Sci. Technol. 9 (1993)

3. M. Boćkowski, B. Łucznik, I. Grzegory, S. Krukowski, M. Wróblewski, S. Porowski, J. Phys.: Condens. Matter, 14 (2002)

4. C. Lin, S. Chung, J. Mater. Res. 16 (2001)

5. A. Wilmański, M. Bućko, Z. Pędzich, J. Szczerba, J. Mater. Sci. Chem. Eng. 2 (2014)

6. T. Khabas, Glass and Ceramics, 59 (2002) 
7. A. Ilyin, L. Root, A. Mostovshchikov, Key Eng. Mat. 685 (2016)

8. A. Mostovshchikov, A. Ilyin, I. Zabrodina, Key Eng. Mat. 685 (2016)

9. A. Il'in, A. Mostovshchikov, L. Root, Tech. Phys. Lett. 37 (2011)

10. A. Il'in, A. Mostovshchikov, N. Timchenko, Combust. Explos. Shock, 49 (2013)

11. A. Il'in, L. Proskurovskaya, Combust. Explos. Shock, 26 (1990)

12. A. Il'in, L. Root, A. Mostovshchikov, Techn. Phys. Lett. 57 (2012)

13. A. Mostovshchikov, A. Ilyin, A. Azanov, I. Egorov Key Eng. Mat. 685 (2016)

14. A. Mostovshchikov, A. Il'in, P. Chumerin, Yu. Yushkov, V. Vaulin, B. Alekseev, Tech. Phys. Lett. 42 (2016)

15. A. Gromov, L. Chukhlomina, Nitride Ceramics: Combustion Synthesis, Properties and Applications, Weinheim: Wiley-VCH (2014)

16. A. Gromov, U. Teipel, Metal Nanopowders: Production, Characterization, and Energetic Applications, Weinheim: Wiley-VCH, (2014)

17. A. Ilyin, A. Gromov, V. Merkulov, A. Mostovshchikov, Russ. Phys. J. 58, 6/2 (2015)

18. A. Il'in, L. Root, Russ. Phys. J. 54, 11/3 (2011) 